\title{
Análisis descriptivo del personal militar español asistido por el Servicio de Psicología del ROLE 2 de Herat, Afganistán
}

\author{
Martínez Sánchez. JA. ${ }^{1}$
}

Sanid. mil. 2012; 68 (3): 163-167; ISSN: 1887-8571

\begin{abstract}
RESUMEN
Introducción: Desde 2005, el Servicio de Psicología del ROLE 2 de la Base de Apoyo Avanzado de Herat (Afganistán) presta asistencia psicológica al personal militar español desplegado en dicha provincia afgana. Esta asistencia adquiere un gran valor para el bienestar de nuestras tropas, dadas las características de riesgo y peligrosidad de las misiones que realizan en Afganistán. Material y métodos: Estudio observacional, descriptivo, transversal del personal atendido por el Servicio de Psicología del ROLE 2 entre mayo de 2005 y septiembre de 2009. Resultados: El número total de casos analizados fue 172. Los trastornos de ansiedad y los trastornos adaptativos fueron las categorías diagnósticas asignadas con mayor frecuencia, siendo la actitud terapéutica más común el control de la ansiedad, la terapia cognitiva y la repatriación o adelanto de la vuelta a territorio nacional. Conclusión: Para favorecer la adaptación de nuestro personal a zona de operaciones es necesario impartir programas formativos y educativos que permitan identificar, prevenir y afrontar estos trastornos: Del mismo modo, es preciso mejorar el perfil profesiográfico de cada individuo en misiones en el exterior.
\end{abstract}

PALABRAS CLAVE: Adaptación psicológica, estrés, zona de operaciones, personal militar, ISAF (International Security Assistance Force), Afganistán.

\section{A descriptive analysis of the Spanish military personnel attended by the Psychology Service at ROLE 2, Herat, Afghanistan SUMMARY}

Introduction: Since 2005, the Service of Psychology of ROLE 2 of the Forward Support Base, Herat (Afghanistan), provides psychological assistance to the Spanish military personnel deployed in this Afghan province. This assistance takes a large value for the welfare of our troops, given the nature of risk and danger of the missions in Afghanistan. Methods: Observational, descriptive, transversal of military people assisted by the Service of Psychology ROLE 2 between May 2005 and September 2009. Results: The total number of cases analyzed was 172. Anxiety disorders and adjustment disorders were the diagnostic categories the more frequently assigned, being the most common therapeutic approach to control anxiety, cognitive therapy and repatriation or return to Spain. Conclusion: Training and educational programs are necessary to promote the adaptation of our troops in operations area in order to identify, prevent and manage these disorders. Similarly, it is necessary to improve the professional profile of each individual in missions abroad.

KEY WORDS: Psychological adjustement, stress, operations area, military troop, ISAF (International Security Assistance Force), Afghanistan.

\section{INTRODUCCIÓN}

El 27 de diciembre de 2001, el Consejo de Ministros autorizó la participación de tropas españolas en la Fuerza Internacional de Asistencia para la Seguridad (International Security Assistance Force, ISAF, en inglés) en apoyo al Gobierno interino afgano. El primer contingente español se desplegó en Afganistán a finales de enero de 2002 y estaba compuesto por unos 350 efectivos. Desde entonces, España ha mantenido una presencia militar activa en aquel país, ajustada a la evolución de las necesidades en materia de seguridad, reconstrucción y desarrollo de la zona ${ }^{1}$. De este modo, a finales de

${ }^{1}$ Cap. Psicólogo. Centro de Psicología de San Fernando (Armada). Cádiz. España.

Dirección para correspondencia: Jefatura de Apoyo Sanitario de la Bahía de Cádiz. Hospital Básico de la Defensa. Paseo Capitán Conforto, s/n. 11110. San Fernando, Cádiz. E-mail: jamartsan@ea.mde.es; juams1968@hotmail.com

Recibido: 29 de diciembre de 2011

Aceptado: 27 de marzo de 2012
2010, cerca de 17.000 militares españoles habían rotado en los distintos relevos desplegados en Afganistán, lo que ha supuesto que el coste económico de nuestra participación en ISAF ascendiera a 2.200 millones de euros ${ }^{2}$.

La asunción por el Ejército del Aire del mando de la Base de Apoyo Avanzado (Forward Support Base, FSB en inglés) «Camp Arena», Herat, el 18 de mayo de 2005 supuso un hito importante en la contribución de nuestro país a las fuerzas de ISAF. La misión principal de la FSB es proporcionar apoyo a los cuatro Equipos de Reconstrucción Provincial (Provincial Reconstruction Team, PRT, en inglés) que la OTAN mantiene desplegados en la región oeste de Afganistán, en concreto los de Farah, Chaghcharan, Herat y Qala-iNaw, a cargo de Estados Unidos, Lituania, Italia y España, respectivamente. Desde entonces hasta la actualidad, la FSB de Herat ha acogido a los numerosos contingentes y relevos españoles y aliados allí desplegados, llegando a albergar hasta un máximo de 1.329 militares, 890 de ellos españoles ${ }^{3}$. Para atender las posibles necesidades sanitarias de este personal y de la población local que lo pudiera requerir, la FSB dispone de un hospital militar de campaña tipo 
ROLE 2 con capacidades y servicios como triage, estabilización, cirugía, cuidados intensivos, evacuación médica, radiodiagnóstico, laboratorio de análisis, farmacia hospitalaria, servicio de odontología y psicología.

Una de las principales aportaciones de la Psicología al ámbito militar es su participación en operaciones en el exterior, con la misión de velar por el cuidado de la salud mental de los contingentes desplegados, a los que presta asistencia, orientación y apoyo psicológico ${ }^{4}$. Para ello, el Servicio de Psicología del destacamento español en "Camp Arena» realiza diversas funciones, entre las que destacan las de asesoramiento técnico al mando para la optimización del funcionamiento del personal y unidades, las labores formativas y preventivas, el apoyo a la selección de personal afgano o la elaboración de informes periciales a solicitud del mando. Sin embargo, al estar encuadrado dentro de una unidad hospitalaria como es el ROLE 2, su función prioritaria es la evaluación, diagnóstico y tratamiento del personal militar español que presenta algún tipo de dificultad de adaptación a la zona de operaciones 5 . Esta labor asistencial cobra gran importancia, dada las especiales características de riesgo y peligrosidad de las misiones que desempeñan nuestras tropas desplegadas en Afganistán.

El presente trabajo se plantea con el objetivo de conocer las características socioprofesionales y asistenciales que definen al personal atendido por el Servicio de Psicología del ROLE 2, característi-

Tabla 1. Distribución de la muestra según la unidad a la que estaban adscritos.

\begin{tabular}{|c|c|c|c|}
\hline Unidad & $\begin{array}{l}\text { Hombres } \\
\text { (n) }\end{array}$ & $\begin{array}{l}\text { Mujeres } \\
\text { (n) }\end{array}$ & Total (\%) \\
\hline ROLE (1 y 2$)$ & 17 & 8 & $25(14,53)$ \\
\hline $\begin{array}{l}\text { Unidad de Helicópteros de Protección } \\
\text { ET (ASPUHEL) }\end{array}$ & 24 & 0 & $24(13,95)$ \\
\hline National Support Element (NSE) & 19 & 1 & $20(11,63)$ \\
\hline $\begin{array}{l}\text { Unidad de Helicópteros EA en ISAF } \\
\text { (HELISAF) }\end{array}$ & 15 & 3 & $18(10,46)$ \\
\hline Quick Reaction Force (QRF) & 15 & 2 & $17(9,88)$ \\
\hline Centro de Comunicaciones (CECOM) & 6 & 2 & $8(4,65)$ \\
\hline $\begin{array}{l}\text { Combined Air Terminal Operations } \\
\text { (CATO) }\end{array}$ & 6 & 0 & $6(3,49)$ \\
\hline Force Protection & 3 & 1 & $4(2,33)$ \\
\hline $\begin{array}{l}\text { Grupo Táctico (Batallón) Apoyo } \\
\text { Elecciones (GTAPOEL) }\end{array}$ & 2 & 1 & $3(1,74)$ \\
\hline Tactical Air Control Party (TACP) & 3 & 0 & $3(1,74)$ \\
\hline Servicio Contraincendios & 2 & 0 & $2(1,16)$ \\
\hline Destacamento Guardia Civil Herat & 1 & 0 & $1(0,58)$ \\
\hline Otras unidades FSB $(*)$ & 6 & 1 & $7(4,07)$ \\
\hline Unidades Qala-i-Naw & 5 & 1 & $6(3,49)$ \\
\hline $\begin{array}{l}\text { Operational Mentoring and Liaison } \\
\text { Team (OMLT) }\end{array}$ & 4 & 0 & $4(2,33)$ \\
\hline $\begin{array}{l}\text { Provincial Reconstruction Team (PRT) } \\
\text { de Kandahar }\end{array}$ & 1 & 0 & $1(0,58)$ \\
\hline Regional Commander West (RCW) & 1 & 0 & $1(0,58)$ \\
\hline Sin datos & 20 & 2 & $22(12,79)$ \\
\hline Total & 150 & 22 & $172(100)$ \\
\hline
\end{tabular}

$(*)$ Incluye Base Operation Center (BOC), CNI, Mess \& Billeting (M\&B) y Destacamento Aéreo de Transporte (ALCOR). cas de interés para el trabajo del personal psicólogo desplegado en zona de operaciones.

\section{MATERIAL Y MÉTODO}

Se realizó un estudio observacional, descriptivo, transversal del personal atendido por el Servicio de Psicología del ROLE 2 (Herat) entre mayo de 2005 ( $1 .^{\circ}$ relevo del Ejército del Aire) y septiembre de 2009 (14. ${ }^{\circ}$ relevo, $1 .^{\text {a }}$ rotación). Para ello se analizaron 172 historiales psicológicos, durante la estancia del autor en zona de operaciones entre los meses de julio y septiembre de 2009.

Se han analizado: a) variables de carácter socioprofesional como sexo, edad, empleo, ejército y unidad de pertenencia; y b) variables asistenciales como vía de remisión, motivo de consulta, antecedentes de trastornos psicológicos, presencia de éstos, categoría diagnóstica asignada o estrategia terapéutica empleada.

Las variables cuantitativas se han descrito mediante medidas de tendencia central y de dispersión: media o mediana y desviación estándar o rango intercuartil (RIC). La descripción de las variables cualitativas se ha realizado mediante frecuencias y porcentajes. El análisis estadístico se ha realizado mediante el programa SPSS, versión 19.

\section{RESULTADOS}

En cuanto a las variables socioprofesionales, un $87,21 \%$ $(n=150)$ son hombres, la media de edad fue 32,31 años, ligeramente superior en el caso de los hombres $(32,38)$ que en el de las mujeres $(29,11)$, y con un RIC 19 a 62 . Un $66 \%$ tenía menos de 40 años y solo 2,91\% eran mayores de 50 años. El 59,09\% de las mujeres atendidas se encontraba en el intervalo de edad 26-30 años. Al analizar las categorías de empleo del personal atendido, un 58,72\% pertenecían a tropa profesional, un $24,42 \%$ a suboficiales y $14,53 \%$ a oficiales; careciéndose de datos sobre la categoría de empleo del $2,33 \%$ restante.

El mayor porcentaje del personal militar atendido pertenecía al Ejército del Aire (43\%), seguido por el personal de Ejército de Tierra $(38,37 \%)$ y de Cuerpos Comunes de las Fuerzas Armadas (7\%). Un sujeto $(0,58 \%)$ pertenecía al destacamento de la Guardia Civil en la FSB de Herat.

Las unidades con mayor porcentaje de personal asistido por el Servicio de Psicología fueron, por este orden, ROLE 1 y 2 (14,53\%), ASPUHEL (13,95\%), NSE (11,6\%), HELISAF $(10,46 \%)$ y la Fuerza de Reacción Rápida, QRF, (9,88\%). También fueron atendidos once militares pertenecientes a unidades ubicadas físicamente en el exterior de la FSB, como los Equipos PRT de Qala-i-Naw y Kandahar, o el Equipo Operativo de Asesoramiento y Enlace (OMLT) de Camp Stone (Tabla 1).

En la tabla 2 se muestran las diferentes vías de remisión del personal que acudió al Servicio de Psicología, siendo las más frecuentes la solicitud por parte de sus mandos directos, la iniciativa propia y la derivación por los servicios médicos de ROLE 2 y ROLE 1.

Un total de 22 militares del contingente español (12,79\%) presentaban antecedentes psicológicos previos de importancia, incluyendo cuatro casos de sujetos diagnosticados de trastorno psicológico en misiones anteriores en el exterior, dos de los cuales hubieron 
Tabla 2. Distribución de la muestra según su vía de remisión.

\begin{tabular}{|lcc|}
\hline Remitido por & sujetos $(\mathbf{n})$ & $\mathbf{\%}$ \\
\hline Mandos & 67 & 38,95 \\
Propia iniciativa & 59 & 34,3 \\
ROLE 2 & 30 & 17,44 \\
ROLE 1 & 6 & 3,49 \\
Páter & 2 & 1,16 \\
Auditor & 1 & 0,58 \\
Compañeros & 1 & 0,58 \\
Sin datos & 6 & 3,49 \\
\hline Total & 172 & 100 \\
\hline
\end{tabular}

de ser repatriados a España entonces. Otros ocho militares habían sido diagnosticados previamente en territorio nacional de algún trastorno psicológico, incluyendo crisis de ansiedad, trastornos por somatización y un caso de intento autolítico.

De los militares estudiados, quince habían estado sometidos a algún tipo de suceso vital estresante de importancia antes de la misión, y que contribuyeron posteriormente a la aparición de dificultades de adaptación en zona. Entre ellos destaca la existencia de problemática familiar y conyugal (separaciones, divorcios y otros problemas de relación) y el fallecimiento reciente de seres queridos (familiares o amigos) antes de comenzar la misión.

Los principales motivos de remisión al Servicio de Psicología fueron la valoración y prevención de secuelas psicológicas tras accidentes, atentados o enfrentamientos con la insurgencia afgana, seguida de la sintomatología ansiosa y la existencia de problemática laboral. Catorce sujetos acudieron a consulta para reconocimiento psicológico (periódico o extraordinario), doce lo hicieron en busca

Tabla 3. Distribución de la muestra en función del motivo de remisión a consulta.

\begin{tabular}{|c|c|c|c|}
\hline Motivo consulta & $\begin{array}{l}\text { Hombres } \\
\text { (n) }\end{array}$ & $\begin{array}{l}\text { Mujeres } \\
\text { (n) }\end{array}$ & Total (\%) \\
\hline $\begin{array}{l}\text { Valoración/prevención secuelas } \\
\text { psicológicas }\end{array}$ & 31 & 2 & $33(19,19)$ \\
\hline Sintomatología ansiedad & 25 & 4 & $29(16,86)$ \\
\hline Problemática laboral & 21 & 2 & $23(13,37)$ \\
\hline $\begin{array}{l}\text { Reconocimiento periódico o } \\
\text { extraordinario }\end{array}$ & 13 & 1 & $14(8,14)$ \\
\hline Consejo psicológico & 9 & 3 & $12(6,98)$ \\
\hline Alteración del sueño & 10 & 0 & $10(5,81)$ \\
\hline Problemática familiar/conyugal & 8 & 1 & $9(5,23)$ \\
\hline Sintomatología depresiva & 4 & 2 & $6(3,49)$ \\
\hline Conducta inapropiada/extraña & 5 & 1 & $6(3,49)$ \\
\hline Sintomatología psicosomática & 4 & 1 & $5(2,91)$ \\
\hline Dolor/cefaléas/malestar general & 5 & 0 & $5(2,91)$ \\
\hline Alteración de conducta & 3 & 1 & $4(2,33)$ \\
\hline Sintomatología ansioso-depresiva & 2 & 1 & $3(1,74)$ \\
\hline Ideación suicida/conducta autolítica & 1 & 1 & $2(1,16)$ \\
\hline Consumo excesivo de alcohol & 2 & 0 & $2(1,16)$ \\
\hline Miedo a estímulos específicos & 1 & 1 & $2(1,16)$ \\
\hline Otros & 6 & 1 & $7(4,07)$ \\
\hline Total & 150 & 22 & $172(100)$ \\
\hline
\end{tabular}

de consejo psicológico de tipo personal y diez presentaban alteraciones del sueño, principalmente insomnio. Nueve militares refirieron algún tipo de problemática familiar y conyugal que dificultaba su adaptación (Tabla 3 ).

En la tabla 4 se aprecia que un 52,91\% $(n=91)$ de los militares españoles evaluados fueron diagnosticados de algún tipo de trastorno psicológico, mientras que el 40,7\% $(\mathrm{n}=70)$ recibió un diagnóstico compatible con la normalidad. De un $6,39 \%(n=11)$ no se pudo realizar un diagnóstico concreto por diversos motivos, como la presencia de síntomas inespecíficos, la escasa colaboración de los sujetos o su resistencia a ser evaluados.

Siguiendo la clasificación de la American Psychological Association $^{6}$, en la tabla 5 se muestran las categorías diagnósticas asignadas a dichos sujetos. Destacan los trastornos de ansiedad (40,65\% de los sujetos diagnosticados) y los trastornos adaptativos (20,88\%). Se dieron seis casos de duelo complicado y de problemática relacionada con el ámbito laboral (6,59\%), y un 5,49\% de los militares evaluados $(\mathrm{n}=5)$ presentó trastornos del sueño. Señalamos también dos casos de consumo excesivo de alcohol y un trastorno psicótico, en concreto, un brote psicótico agudo.

Un 25,43\% del personal atendido no requirió ningún tratamiento; en cambio un $19,08 \%$ requirió su repatriación o adelanto de la vuelta a territorio nacional, un $16,76 \%$ recibió entrenamiento en técnicas de control de ansiedad (relajación muscular, respiración profunda), y a un 13,87\% de los militares asistidos se les aplicó técnicas cognitivas de modificación de pensamiento. Se aplicaron

Tabla 4. Distribución de la muestra en función de la presencia de trastornos psicológicos.

\begin{tabular}{|lccc|}
\hline & $\begin{array}{c}\text { Hombres } \\
\mathbf{n}(\mathbf{\%})\end{array}$ & $\begin{array}{c}\text { Mujeres } \\
\mathbf{n}(\mathbf{\%})\end{array}$ & $\begin{array}{c}\text { Total } \\
\mathbf{n}(\%)\end{array}$ \\
\hline Normalidad/apto reconocimiento & $63(36,63)$ & $7(4,07)$ & $70(40,7)$ \\
Presencia trastorno & $77(44,77)$ & $14(8,14)$ & $91(52,91)$ \\
Sin diagnóstico específico & $10(5,81)$ & $1(0,58)$ & $11(6,39)$ \\
\hline Total & $150(87,21)$ & $22(12,79)$ & $172(100)$ \\
\hline
\end{tabular}

Tabla 5. Distribución del personal español según categorías diagnósticas asignadas.

\begin{tabular}{|lccc|}
\hline Categoría diagnóstica & $\begin{array}{c}\text { Hombres } \\
\mathbf{n}(\mathbf{\%})\end{array}$ & $\begin{array}{c}\text { Mujeres } \\
\mathbf{n}(\mathbf{\%})\end{array}$ & $\begin{array}{c}\text { Total } \\
\mathbf{p ~ ( \% )}\end{array}$ \\
\hline Trastornos de ansiedad & $32(35,16)$ & $5(5,49)$ & $37(40,65)$ \\
Trastornos adaptativos & $16(17,58)$ & $3(3,3)$ & $19(20,88)$ \\
Duelo complicado & $5(5,49)$ & $1(1,1)$ & $6(6,59)$ \\
Problemática laboral & $5(5,49)$ & $1(1,1)$ & $6(6,59)$ \\
Trastornos del sueño & $5(5,49)$ & 0 & $5(5,49)$ \\
Reacción adaptativa & $3(3,29)$ & $2(2,2)$ & $5(5,49)$ \\
Problemática conyugal/familiar & $3(3,29)$ & $1(1,1)$ & $4(4,39)$ \\
Trastornos del estado de ánimo & $2(2,2)$ & 0 & $2(2,2)$ \\
Trastornos por consumo alcohol & $2(2,2)$ & 0 & $2(2,2)$ \\
Rasgos de personalidad & $2(2,2)$ & 0 & $2(2,2)$ \\
psicopatológica & 0 & $1(1,1)$ & $1(1,1)$ \\
Trastornos psicóticos & $1(1,1)$ & 0 & $1(1,1)$ \\
Trastornos somatomorfos & $1(1,1)$ & 0 & $1(1,1)$ \\
Trastornos control impulso & $77(84,62)$ & $14(15,38)$ & $91(100)$ \\
\hline Total & & &
\end{tabular}


Tabla 6. Estrategias terapéuticas aplicadas al personal español de ISAF atendido por el Servicio de Psicología del ROLE 2.

\begin{tabular}{|c|c|c|c|}
\hline Pautas terapéuticas & $\begin{array}{l}\text { Hombres } \\
\text { n }\end{array}$ & $\begin{array}{l}\text { Mujeres } \\
\mathbf{n}\end{array}$ & $\begin{array}{c}\text { Total n } \\
(\%)\end{array}$ \\
\hline No requirió tratamiento & 41 & 3 & $44(25,43)$ \\
\hline $\begin{array}{l}\text { Repatriación/Adelanto vuelta a } \\
\text { territorio nacional }\end{array}$ & 28 & 5 & $33(19,08)$ \\
\hline $\begin{array}{l}\text { Control ansiedad (relajación, } \\
\text { respiración) }\end{array}$ & 23 & 6 & $29(16,76)$ \\
\hline $\begin{array}{l}\text { Terapia cognitiva (detención, } \\
\text { distracción, reestructuración, } \\
\text { visualización) }\end{array}$ & 18 & 6 & $24(13,87)$ \\
\hline Higiene de sueño & 13 & 3 & $16(9,25)$ \\
\hline Psicofarmacología & 9 & 1 & $10(5,78)$ \\
\hline Reforzamiento habilidades sociales & 8 & 2 & $10(5,78)$ \\
\hline Retirada de armamento & 5 & 3 & $8(4,62)$ \\
\hline Técnicas de modificación de conducta & 7 & 0 & $7(4,05)$ \\
\hline $\begin{array}{l}\text { Medidas de ajuste laboral (cambio de } \\
\text { turno, destino, corimec) }\end{array}$ & 5 & 2 & $7(4,05)$ \\
\hline Consejo psicológico & 3 & 3 & $6(3,47)$ \\
\hline Técnicas afrontamiento estrés & 4 & 1 & $5(2,89)$ \\
\hline $\begin{array}{l}\text { Prevención Trastorno por Estrés } \\
\text { Postraumático }\end{array}$ & 5 & 0 & $5(2,89)$ \\
\hline Elaboración duelo & 3 & 0 & $3(1,73)$ \\
\hline Recomendación baja en vuelo & 2 & 0 & $2(1,16)$ \\
\hline Programa de reducción bebida & 0 & 1 & $1(0,58)$ \\
\hline Total & 174 & 36 & 210 \\
\hline
\end{tabular}

pautas para una correcta higiene de sueño a un 9,25\% de los militares asistidos y un 5,78\% de los casos requirió tratamiento psicofarmacológico dirigido por el personal médico del ROLE. En la tabla 6 se muestran estos datos, considerando que el personal atendido podía requerir más de una las técnicas señaladas.

\section{DISCUSIÓN}

Al analizar la distribución por sexos del personal asistido, podría inferirse que el porcentaje de personal femenino asistido (12,79\%) se corresponde con el existente en nuestras FAS, que durante el periodo comprendido entre 2005 y 2009 osciló -según fuentes oficiales- entre el $11,5 \%$ y $12,2 \%$, respectivamente ${ }^{7}$. No obstante, dicho porcentaje es notablemente superior al porcentaje de participación de mujeres militares españolas en operaciones de mantenimiento de paz, que se sitúa en el $6 \%$.

La alta proporción de personal del Ejército de Tierra atendido por el Servicio de Psicología se encuentra en relación con la distribución del contingente militar español en ISAF, a la que dicho Ejército aporta un contingente importante. En este sentido, el hecho de que el Servicio de Psicología del ROLE 2 de la FSB de Herat pertenezca al Ejército del Aire y esté formado por facultativos adscritos a él obliga a establecer los adecuados cauces de comunicación y coordinación con los Servicios de Psicología del Ejército de Tierra, a efectos de obtención de información relacionada con los expedientes psicológicos de su personal desplegado en zona, incluyendo datos sobre aptitud psicofísica, resultados de reconocimientos psicoló- gicos previos y existencia de posibles antecedentes. Por otra parte, la mínima proporción de personal de la Guardia Civil atendido en el ROLE 2 por motivos psicológicos se encuentra en consonancia con la escasa presencia de miembros de este cuerpo durante las fechas que comprende este estudio, tanto en la misión de ISAF en general, como en la provincia de Herat en particular.

En cuanto a las unidades que demandan mayor asistencia psicológica, ROLE 1 y 2 son las unidades a las que pertenecen el mayor porcentaje de militares españoles asistido por el Servicio de Psicología, lo que posiblemente se deba a dos factores. En primer lugar, a los altos niveles de estrés que soportan estos profesionales y que se relacionan con el denominado síndrome de burnout ${ }^{9}$; y en segundo lugar, a que la ubicación física del Servicio de Psicología dentro del ROLE permite al resto de personal sanitario una mayor facilidad y disponibilidad para acudir a él en caso de requerir asistencia, apoyo o consejo psicológico. Por otro lado, la presencia de un alto porcentaje de personal asistido pertenecientes a las unidades de helicópteros del Ejército del Aire (HELISAF) y Tierra (ASPUHEL) y la Fuerza de Reacción Rápida (Quick Reaction Force, QRF, en inglés), parece relacionarse con la especial peligrosidad y riesgo de las misiones y funciones que realizan y la consiguiente vulnerabilidad de sus miembros a padecer desórdenes psicológicos. A este respecto, el hecho de que la Base de Apoyo Provincial Base de Apoyo Provincial (Provincial Suppot Base, POB, en inglés) española de Qala-i-Naw disponga desde 2010 de Servicio de Psicología propio redunda, sin dudas, en un mayor bienestar y adaptación psicológica de nuestras tropas allí desplegadas, incluyendo el personal de la QRF.

Puesto que entre las principales vías de remisión del personal español asistido se encuentran los mandos y la propia iniciativa, seguida de ROLE 2 y 1, el autor sugiere: a) la necesidad de que tanto el personal de las distintas unidades como sus mandos directos conozcan la labor preventiva, asistencial y de apoyo que realizan los servicios de Psicología desplazados en misiones en el exterior. A ello puede contribuir un aumento de la participación del personal psicólogo en las sesiones de in-processing que se imparten al personal que se incorpora a zona de operaciones; b) la importancia de facilitar asistencia psicológica directa e inmediata al personal que lo demande, minimizando los trámites burocráticos y posibles filtros por parte de los mandos directos de unidades; y c) la coordinación directa del psicólogo en zona de operaciones con los servicios médicos del ROLE 2, especialmente con el personal de triage y enfermería, para la derivación al Servicio de Psicología de todo aquel personal con sintomatología desadaptativa. En el mismo sentido, y dado que no es infrecuente asistir a personal perteneciente a unidades externas a la FSB, se recomienda una mayor coordinación directa con el personal médico (ROLE 1) y con los mandos de dichas unidades, al objeto de prevenir y detectar la presencia de sujetos con sintomatología desadaptada o psicopatológica.

Otro aspecto importante es la presencia de antecedentes psicológicos en un porcentaje relevante de los militares asistidos por el Servicio de Psicología, lo que obliga a prestar mayor atención a la evaluación de dichos antecedentes en los reconocimientos psicológicos premisión, mejorando de esta manera los procesos de selección y evitando así un porcentaje importante de posteriores repatriaciones a territorio nacional. Estas repatriaciones tienen a menudo un coste añadido, no sólo por el aspecto económico que acarrean sino también porque suponen un aumento de carga de trabajo para el personal que permanece en zona de operaciones y se ve obligado a 
realizar las tareas del repatriado hasta la llegada de su relevo. No podemos olvidar tampoco el coste personal, psicológico y emocional que implica para aquellos reservas que deben ser movilizados para sustituir al personal repatriado. Por tanto, es fundamental afinar los reconocimientos premisión para detectar y filtrar a aquellos sujetos con antecedentes personales y psicológicos que pudieran condicionar y dificultar su posterior adaptación a la misión. A este respecto, merecen especial consideración los casos de personal ya repatriado en misiones anteriores y aquellos otros con diagnóstico psicológico previo. También se hace necesario evaluar cuidadosamente aspectos como la existencia de problemática familiar o conyugal (separación, divorcio, enfermedad graves de familiares cercanos, etc.), ya que la experiencia en zona de operaciones indica que probabilidad de presentar conductas inadaptadas en zona de operaciones aumenta en el caso de sujetos con dificultades conyugales o familiares graves ${ }^{10}$.

Los principales motivos de remisión al Servicio de Psicología de Herat son muy similares a los obtenidos en otros estudios e investigaciones realizadas por la Unidad de Psicología de la Dirección de Sanidad del Ejército de Tierra, que muestran la incidencia en zona de operaciones de los trastornos de estrés/ansiedad, trastornos de adaptación y problemática, tanto profesional como laboral ${ }^{11}$. En base a esta casuística, y a semejanza de lo realizado por otros ejércitos aliados -principalmente los de los Estados Unidos y Reino Unido-, consideramos fundamental instaurar programas de entrenamiento psicológico que aumenten la resiliencia de nuestros militares, entendida ésta como la capacidad de soportar y superar las adversidades, para de esta manera mejorar su actuación en situaciones de estrés y prevenir la aparición de trastornos psicológicos ${ }^{12}$.

Finalmente, de cara a una mejor adaptación de nuestro personal es importante también lograr un buen ajuste al destino y puesto que van a ocupar en zona de operaciones. A ello deben contribuir una correcta elaboración de los perfiles profesiográficos de estos puestos ${ }^{11}$, así como la realización de estudios de clima y satisfacción laboral en aquellas unidades con mayor incidencia de desajustes y conductas desadaptativas.

\section{CONCLUSIONES}

1. Para mejorar la adaptación del personal desplegado en el exterior es necesaria, por parte de los Servicios de Psicología, la difusión de programas formativos y educativos al objeto de identificar, prevenir y afrontar la aparición de trastornos psicológicos, máxime en unidades de mayor vulnerabilidad como las de helicópteros (HELISAF y ASPUHEL) y QRF.

2. Es importante una mayor coordinación entre los Servicios de Psicología de los distintos ejércitos para la elaboración de los adecuados perfiles profesiográficos que posibiliten una mejor asignación del personal a los distintos puestos, funciones y misiones en operaciones en el exterior.

\section{AGRADECIMIENTOS}

El autor quiere expresar su agradecimiento a todo el personal psicólogo desplegado en el Servicio de Psicología del Destacamento de ISAF en «Camp Arena», Afganistán, por haber contribuido con su trabajo a la elaboración de este estudio. Agradecer, igualmente, al personal de la Unidad de Psicología de la Dirección de Sanidad del Ejército del Aire su apoyo y asistencia técnica durante el despliegue del autor en el ROLE 2 de Herat.

\section{BIBLIOGRAFÍA}

1. Ministerio de Defensa. ISAF. Disponible en: http://www.defensa.gob.es/areasTematicas/misiones/enCurso/misiones/mision_03.html

2. Real Instituto Elcano: ABC de la misión en Afganistán. En: http://www.realinstitutoelcano.org/wps/portal/rielcano/MisionesDePaz/Afganistan

3. Ejército del Aire. ISAF. FSB (Herat). Disponible en: http://www.ejercitodelaire. mde.es/ea/pag?idDoc $=$ F21D0D4745FC2245C125746E002E2FD3\&idRef $=$ C7A 231AFA8AA7F47C12574750020962D

4. Albarracín, D. El carácter sanitario de la Psicología en el ámbito militar. Entrevista al Jefe de la Unidad de Psicología de la IGESAN. INFOCOP 2008; 39: 21-24.

5. Martín, G. El Servicio de Psicología del Ejército del Aire en misiones fuera de territorio nacional. Revista de Aeronáutica y Astronáutica 2010; 798: 976-978.

6. American Psychological Association. Manual diagnostico y estadístico de los trastornos mentales: texto revisado. Barcelona: Masson, 2005.

7. Observatorio Militar para la Igualdad. Datos sobre el personal en el Ministerio de Defensa (2011). En: http://www.defensa.gob.es/Galerias/areasTematicas/observatorio/fichero/estadisticas/WEB_DATOS_2011.pdf

8. Del Val, C. La mujer militar en las misiones de paz de la Unión Europea y las Naciones Unidas. En: Gómez Escarda M e Sepúlveda I (eds.). Las mujeres militares en España (1988-2008). Madrid, Instituto Universitario «General Gutiérrez Mellado», 2009, pp. 201-218.

9. Cáceres, G. G., Villanueva, R., Méndez, J. R, García, M.V. y Domínguez, V. Prevalencia del Síndrome de en médicos y enfermeros de un hospital militar. Sanidad Militar 2009; 65 (2): 83-94.

10. Headquarters Department of the Army. Combat Stress, FM 6-22-5. Washington, DC, 2000: 29.

11. Robles, J I. La Psicología y el combatiente en los conflictos actuales. Conferencia impartida en las Jornadas sobre Sanidad Militar en la Real Academia Nacional de Medicina. Madrid, 18 de noviembre de 2010.

12. García Silgo, M y Bardera, P. Resiliencia: la Defensa Psicológica. Revista Española de Defensa 2010; 266: 54-55. 